\title{
La gestión del ciclo del agua a través de una investigación- acción participada. El caso de las Hortes de Baix
}

\author{
Elena Albareda Fernández ${ }^{1}$, Marta Serra Permanyer ${ }^{2}$ \\ CICLICA [space, community and ecology] 1,2, Universitat Politècnica de Catalunya UPC-Barcelona Tech 1,2 \\ elena.albareda@ciclica.eu,marta.serra-permanyer@upc.edu
}

\begin{abstract}
Resumen. Esta experiencia trata de recuperar un espacio de huertas de regadío históricas situadas en la región metropolitana de Barcelona. Partiendo de comprender la gestión tradicional del agua como patrimonio, el proyecto tiene un doble objetivo: por un lado, promueve el diseño urbano sostenible y, por el otro, favorece una nueva consciencia comunitaria sobre el territorio. Las distintas fases tienen la misión de resolver un doble conflicto ambiental relacionado con las aguas de riego: por un lado por la falta de disponibilidad de agua suficiente, y por el otro por el riesgo sanitario que suponen los vertidos contaminantes de aguas negras del centro urbano a la acequia principal de este espacio. Consecuentemente la principal propuesta se basa en la captación de las diversas fuentes de agua limpia termal sobrante de balnearios y lavaderos que no se reutilizan en las huertas actualmente y que permitirán un abastecimiento de agua de riego suficiente y de buena calidad. Mediante un proceso de investigación-acción es posible recuperar la capacidad activa del ciudadano urbano como agente productor, las prácticas cotidianas asociadas con la autogestión del agua como imaginario identitario del lugar, es decir, el patrimonio intangible de la gestión del agua como modelo de referencia hacia la sostenibilidad.
\end{abstract}

Palabras clave: urbanismo socio-ecológico, paisaje cultural, patrimonio intangible, ciclo del agua, investigación-acción participada, metabolismo social.

\begin{abstract}
This experience aims to recover a space of historical irrigated gardens located in the metropolitan area of Barcelona, Spain. From understanding the traditional water management as cultural heritage, the project has a double goal: on the one hand, to promote sustainable urban design and, on the other hand, to boost a new community consciousness connected to the territory. Through several phases, the project solves a double environmental conflict linked to the irrigation system: on one side, the lack of enough water availability and, on the other side, the health risk due to the spill of urban polluted waters in the main irrigation canal of this space. Consequently, the main proposal is based on the gathering of thermal clean water surplus of spas and public washing places -that are not reused in the gardens presently- to provide enough quality water for the irrigation. By means of an action-research process it's possible to recover the active ability of urban citizens as producer agents, as well as the quotidian practices related to water self-management as the imaginary of place identity, that is to say, the intangible heritage of the water management as a reference model towards the sustainability.
\end{abstract}

Keywords: socioecological urbanism, cultural landscape, intangible heritage, water cycle, participatory action-research, social metabolism.

\section{Introducción}

El agua es vida, y los paisajes que genera su gestión social y productiva son la expresión espacial de este hecho cultural a lo largo del tiempo. En la ciudad tradicional, el agua vehiculaba la mayor parte de las actividades urbanas y periurbanas, permitiendo leer la ciudad en relación con su territorio. Una relación basada en un modelo productivo orgánico (Wrigley 1987) donde el ciudadano tenía un papel activo como usuario del agua, mediante prácticas comunitarias cotidianas de autogestión y producción que adaptaban la demanda de este recurso a la oferta disponible en su territorio. El agua era un bien público, los ciudadanos te- 
nían conocimiento activo de cómo se gestionaba a la vez que disfrutaban de un recurso lúdico y social, un bien cultural.

\subsection{Marco teórico}

A partir de la revolución industrial el crecimiento de los sistemas urbanos y su desvinculación con el territorio (Naredo and Valero 1999) ha condicionado también nuestra relación con el agua. Su gestión ha pasado de las comunidades que hacían un uso directo de ella, al modelo municipal, para acabar finalmente privatizada (Guardia et al. 2011). Este hecho ha favorecido la desvinculación entre el agua y sus usuarios, que han adoptado un papel pasivo de consumidor del recurso que, junto al proceso de crecimiento económico, ha transformado el modelo de gestión hacia un sistema basado en la demanda. Las infraestructuras tradicionales han quedado obsoletas, cayendo en el olvido en un proceso de desapropiación. Se ha "robado" el agua a la comunidad, privándole de su capacidad de decisión y favoreciendo la pérdida de sentimiento de comunidad (Hardy and Ward 1984).

Esta desvinculación de la gestión del agua y por lo tanto, de capacidad de decisión sobre su uso no sólo ha alejado a la comunidad del recuso a nivel social o cultural, sino que ha conllevado, junto con el sistema productivo industrializado, a la contaminación frecuente de su ciclo generando impactos ambientales a menudo difíciles de detectar y gestionar por la falta de conocimiento de los ciudadanos. Tanto la consciencia de protección del patrimonio arquitectónico (Choay 1992), como la demanda social de protección ambiental de los sistemas naturales han intentado reaccionar a estos hechos dando cobijo a elementos aislados del ciclo del agua como pueden ser sus infraestructuras físicas (molinos, balsas, acequias) o los espacios naturales receptores de las aguas urbanas (lechos de ríos, humedales). Pero si se comprende la gestión del agua desde una óptica sistémica e integrada es posible leer su gestión tradicional como patrimonio intangible, un patrimonio, que más allá de la "piedra" reconozca el conocimiento tradicional y los dispositivos físicos, culturales, sociales y económicos que permitían la gestión orgánica de los recursos (Laureano 1995) como un modelo de gestión clave hacia la transformación sostenible de nuestro actual metabolismo social urbano (Fischer-Kowalski and Hüttler 1999).

En este sentido, la gestión del agua para el riego de un espacio de huertas periurbanas plantea la extensión de ese patrimonio intangible a la propia actividad agrícola. Una actividad de tradición milenaria en muchos espacios urbanos y periurbanos y que además garantiza el sustento alimentario de muchas familias aportando un cierto grado de autosuficiencia alimentaria local a la ciudad. Los productos hortícolas de proximidad producidos en estos espacios no sólo destacan por la trazabilidad de su producción como garantía de calidad sino que favorecen la preservación del patrimonio cultural de las prácticas cotidianas, autogestionadas de sus productores hortelanos así como su conocimiento tradicional (Vidal and Prades 2013).

Pero intervenir sobre el patrimonio de la gestión del agua y los espacios agrícolas no es posible sin intervenir desde la reactivación de la comunidad. Recuperar el papel proactivo de los agentes que intervienen desde la gestión de los recursos abre la oportunidad de recuperar la memoria pública (Hayden 1995). Una memoria transmitida mediante el relato oral del recuerdo histórico de las prácticas cotidianas compartidas en un espacio común, transmisora del conocimiento tradicional de la gestión del agua y su uso para el riego de los huertos productivos de autoconsumo. 


\subsection{Marco local}

El proyecto se sitúa en el área metropolitana de Barcelona, en la localidad de Caldes de Montbui situada en la denominada Plana del Vallès, territorio emplazado en la depresión pre-litoral, a unos treinta minutos de la costa. Concretamente esta localidad da nombre a uno de los afluentes del río Besòs, la Riera de Caldes. Caldes recibe las aguas procedentes de la cuenca de la sierra pre-litoral pero además se le suman las aguas de otro recurso muy particular: las aguas termales. El pueblo está cruzado de oeste a este por una falla tectónica que da lugar a numerosas surgencias de agua hipertermal $\left(74^{\circ} \mathrm{C}\right)$, el agua más caliente de toda la península ibérica. Desde la época romana, período en que se fundó la localidad con el nombre de Aquae Calidae, buena parte de las tierras de su cuenca se han dedicado al cultivo. Los excedentes de agua termal, ya desde entonces, posibilitaron el riego más allá de las épocas de sequía pluvial.

Consultando fuentes históricas, hemos podido detectar que la zona de huertas donde intervenimos se caracteriza, por tradición, por su alto grado de producción debido a las propiedades del agua termal. En la siguiente imagen podemos distinguir el casco antiguo de la localidad en relación a la superficie de huertas que le abastece, ambas casi con la misma superficie. En la imagen también se detallan las fuentes termales y casas de baño particulares, más los torrentes que van a parar al río.

Pero en la actualidad, Caldes de Montbui, con una población de unos 17.000 habitantes, se caracteriza progresivamente por un modelo de ciudad centrado en el turismo termal y el contacto con el medio agroforestal que lo distingue de Barcelona. El desarrollo urbanístico más la gestión balnearia fortalecida por la ley de minas, ha potenciado la privatización de este recurso relegando las huertas a un mero espacio receptor de sobrantes termales en el mejor de los casos.

A través de la investigación hemos podido detectar que la falta de agua termal en las huertas hoy día no responde a una escasez de agua termal sino a una gestión inadecuada de este recurso. Actualmente, de los $1.000 \mathrm{~m}^{3}$ que afloran diariamente de forma natural, casi dos tercios del total son vertidos al río una vez se han usado en bañeras de hidromasaje y tratamientos, sin contemplar espacios de retención, compensación o enfriamiento.

Si además le añadimos la contaminación de un antiguo torrente que cruza las huertas convertido en cloaca abierta, las posibilidades de riego quedan reducidas al régimen pluvial torrencial de una riera que año tras año reduce su caudal ecológico.

Si observamos la estructura espacial actual del lugar podemos detectar aún espacios ligadas a los cursos del agua que desvelan su memoria: huertas informales, lavaderos frecuentados, acequias, molinos y balsas son huellas aún presentes donde el agua se fundía con los espacios de sociabilidad. Ante ello nos preguntamos ¿Cómo introducir la memoria como parte íntegra de un proceso de proyecto? ¿Cómo llegar al ciudadano para comprender el ciclo del agua a través de la memoria de estos espacios? ¿Cómo activar un proyecto que integre estos espacios productivos con el ciclo del agua de lluvia y termal? ¿Cómo asegurar el riego desde un sistema eficiente, sano y sostenible? 


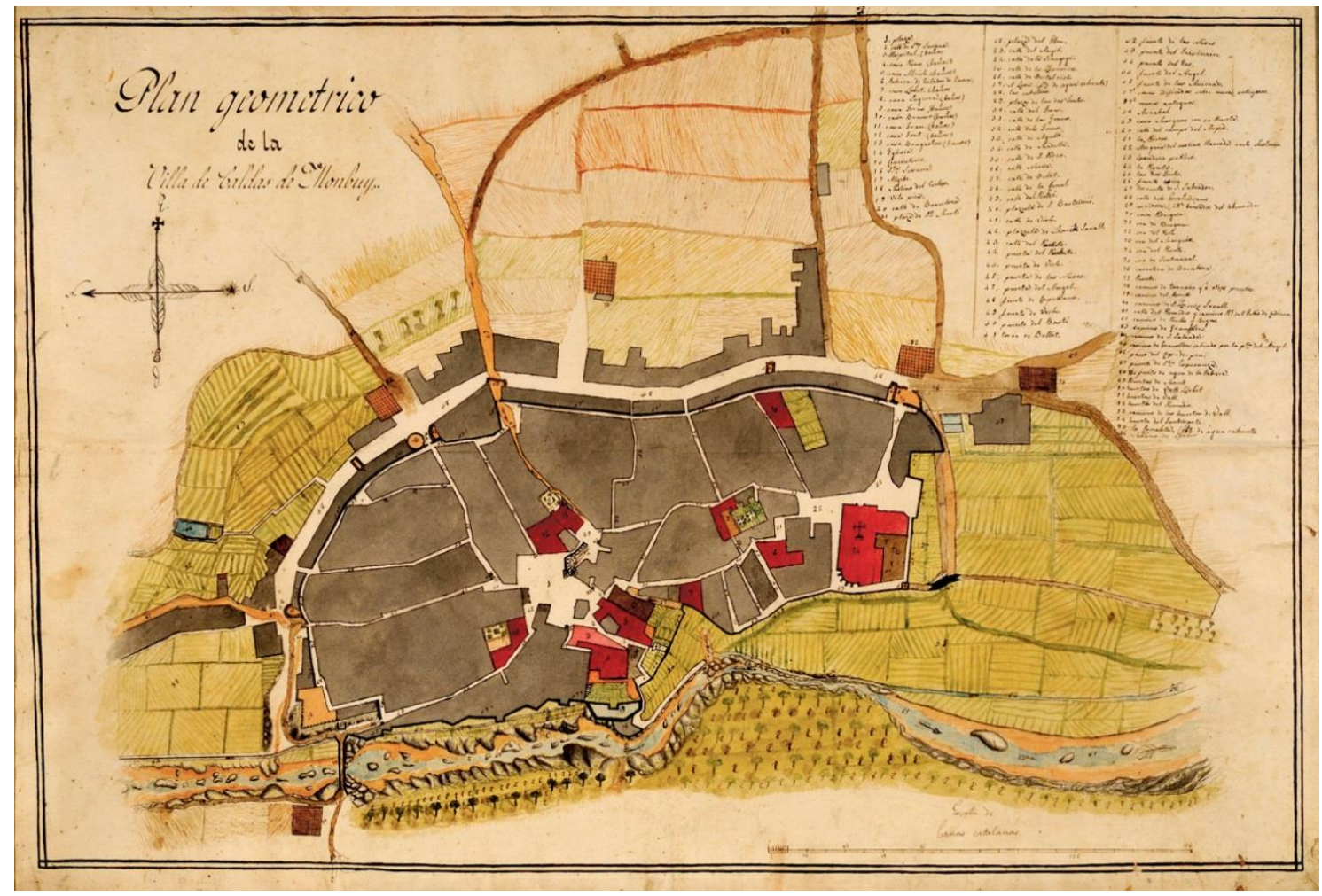

Fig. 1. Modelo de ciudad orgánica, abasteciéndose de su entorno. Plano de la localidad y huertas, fecha no precisa (siglo XVII). Fuente: Pere Maspons i Cadafalch.

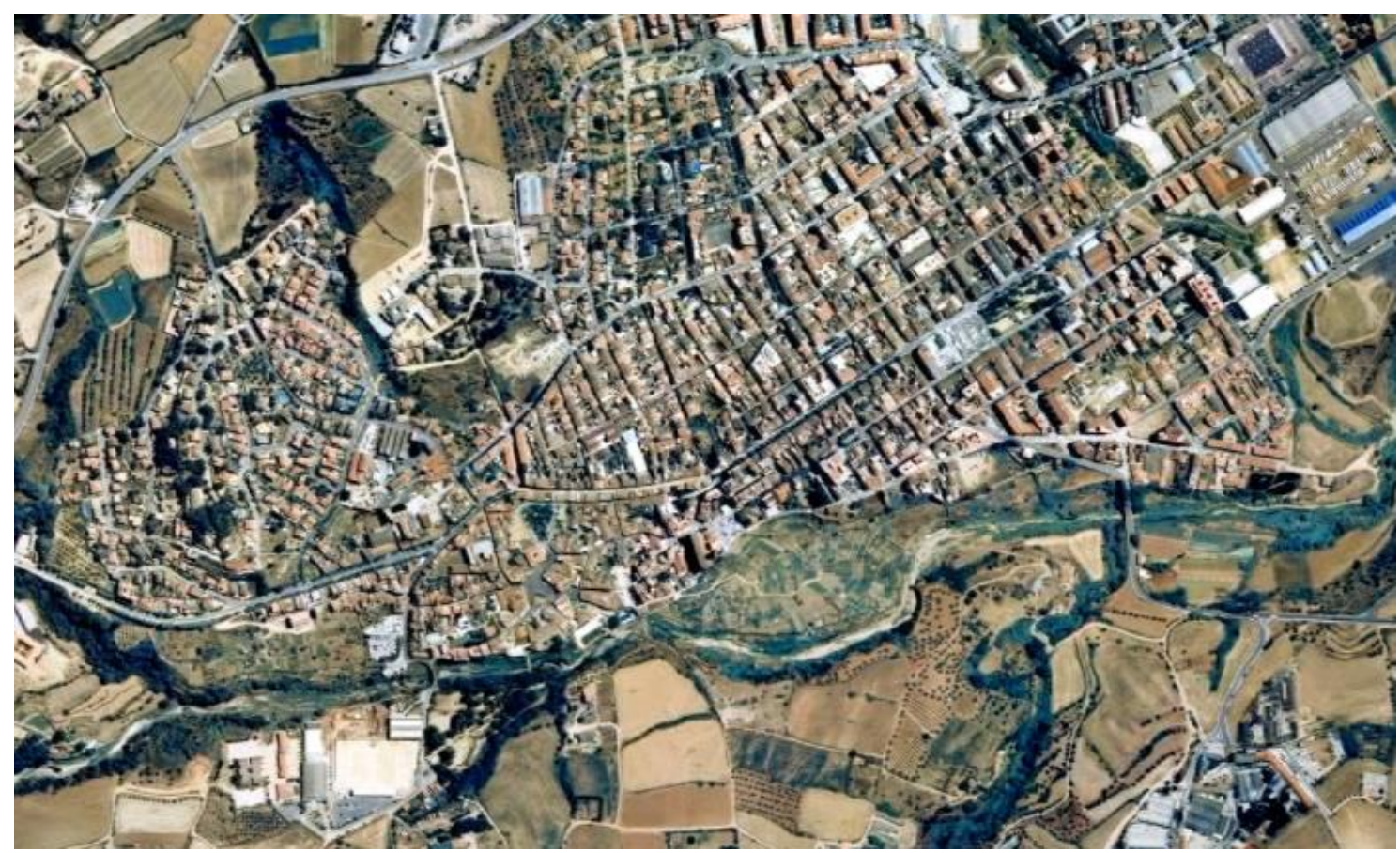

Fig. 2. Modelo de ciudad no orgánica, desvinculada de su entorno. Foto aérea de la localidad a inicios del s. XXI. Fuente: Institut Cartogràfic de Catalunya. 


\section{Detonantes del proyecto}

Nuestra incursión al lugar como técnicas se ha dado de forma gradual. Distintos factores han contribuido a activar otras dinámicas comunitarias catalizadoras de un cambio de mirada y han facilitado una base común para el cambio de visión progresiva. Algunos antecedentes y la detección de un conflicto ambiental han motivado el objeto de proyecto.

\subsection{Antecedentes}

Uno de los principales antecedentes activadores de este proyecto es la Muestra Internacional de Arte Urbano que existe en Caldes de Montbui. Organizado por una institución local, el Museu Thermalia, este evento pone en relieve el potencial de los espacios públicos del casco antiguo ligados con el agua termal, descontextualizándolos y abriendolos a intervenciones artísticas que acentúan su singularidad, re-piensan su uso y reivindican su condición pública (Ferreres et al. 2010). Otra investigación paralela en relación a la reutilización de los lavaderos infrautilizados fue la propuesta Re-thinking Public Space (Serra, Altés 2010), una breve etnografía donde se entrevistaba distintos agentes opinando sobre el futuro de un lavadero abandonado. Por otra parte, la investigación al lugar desde el ámbito universitario desde el Máster en Sostenibilidad de la UPC-Barcelona Tech permitió organizar mesas redondas y de discusión entre los agentes vinculados con el agua termal (balnearios, hortelanos, instituciones, ciudadanos) con el fin de incentivar un nuevo modelo productivo y de gestión, proponiendo entre otros, una línea de acción participativa para integrar el patrimonio intangible en la agenda cultural existente de la localidad.

Aunque en algún caso las propuestas han quedado en el plano teórico, los esfuerzos y éxito popular para la revalorización del patrimonio cultural del agua se han traducido, por ejemplo, en la rehabilitación de un antiguo lavadero abandonado como espacio público para el baño colectivo termal, poniendo énfasis en la condición pública del agua termal de esta localidad. Ha favorecido también que desde la administración municipal se constituya una comisión de trabajo participativa y vinculante llamada 'mesa de espacio público', con implicación de los agentes locales, para gestionar e intervenir de forma consensuada en el espacio público.

En resumen, derivas periurbanas, prácticas espaciales creativas y propuestas de participación ponen de manifiesto el potencial de la gestión comunitaria del agua como bien cultural y eje de transformación social en clave metabólica, favoreciendo un cambio de conciencia que valore el desarrollo económico desde la recuperación de la gestión tradicional del agua, la producción local y la autosuficiencia para una comunidad soberana a la hora de gestionar sus propios recursos.

\subsection{Detección del conflicto}

Más allá de estas intervenciones previas sobre el espacio de las huertas, lavaderos y balnearios y la recuperación de la memoria patrimonial del uso del agua termal en Caldes de Montbui, en los últimos años se han ido acumulando varios factores que han permitido la detección de un claro conflicto ambiental y de gestión del agua en las Hortes de Baix. El vertido sistemático de aguas negras a la acequia principal de abastecimiento de riego de las huertas y su posterior vertido al lecho del curso fluvial natural de la Riera de Caldes ha de- 
tonado la alarma. Una denuncia de sanidad del grupo de ecologistas locales de Gallecs acerca del riesgo para la salud de ingerir hortalizas regadas con aguas fecales y las quejas de hortelanos acerca de la calidad insalubre del agua de riego y la falta de disponibilidad de agua limpia han visibilizado el vertido contaminante de las aguas negras de todo el centro urbano al espacio de huertas patrimoniales. Este factor, junto con los accidentes frecuentes en el mal acceso por encima de los muros de la acequia a las huertas -con la muerte de una hortelana- han favorecido la concienciación definitiva del Ayuntamiento acerca la necesidad de intervenir en el espacio de las huertas y mejorar el abastecimiento y la gestión del agua de riego así como su accesibilidad.

\section{Propuesta}

El objetivo general del proyecto es revalorizar el agua como patrimonio cultural intangible recuperando prácticas asociadas a sus usos de gestión cotidiana para superar los conflictos ambientales actuales. Reconocer el potencial de estos valores permite plantear la reinterpretación de este patrimonio social como motor de transformación espacial sostenibilista (WCED 1987). Partiendo de la comprensión del paisaje como la percepción sociocultural de la forma del territorio (Roger 2000), del modelo de gestión de los recursos, y recuperando el patrimonio de la gestión tradicional del agua desde sus usos, es posible favorecer el cambio de visión necesario para promover un nuevo urbanismo ecológico. Una visión que pretende concretarse en este proyecto en un instrumento clave hacia el nuevo paradigma de la sostenibilidad a través de dos objetivos concretos.

\subsection{Objetivos}

El primer objetivo es favorecer una nueva conciencia ciudadana productiva en relación con la gestión del espacio urbano que habita y el territorio en el que se relaciona. Para ello, más allá de los dispositivos convencionales del urbanismo, existen prácticas informales "bottom-up" que implican a los ciudadanos en la definición del uso del espacio (Certeau 1980). En los espacios excluidos es donde este potencial cobra mayor valor. Centrarse en los espacios intermedios y periurbanos nos permite detectar dinámicas de la historia oral asociadas a los usos informales del agua. Los espacios de sociabilidad derivados de estas prácticas fomentan la capacidad micro-política del individuo en lo que actualmente viene a llamarse urbanismo táctico (Lefebvre 1978) permitiendo la gestión comunitaria en la secuencia funcional de su ciclo (arroyos, acequias, molinos, lavaderos, huertas, balsas, ríos). Intervenir de forma creativa en tales estructuras físicas desde la recuperación de la memoria pública resulta fundamental para reconocer sus prácticas socio-culturales a las que dan cobijo (abastecer, moler, lavar, cultivar, regar, bañarse, alimentar).

El segundo objetivo es promover un diseño urbano sostenible desde la comprensión de la gestión tradicional de los recursos -en este caso del agua termal para riego y de la materia orgánica para la producción de alimentos y la absorción de residuos como compost- desde el conocimiento y las posibilidades técnicas actuales para responder a las necesidades sociales y ambientales. Recuperar el agua termal sobrante de los balnearios, favorecer la gestión del agua por gravedad, separar las distintas calidades de agua para tratamientos y usos diferenciados, son algunas de las líneas de trabajo con las que opera el diseño urbano sostenible para las Hortes de Baix con el objetivo de intervenir en el espacio mediante estrategias 
de ecología urbana basadas en la gestión de los recursos (Bettini et al. 1996) que moviliza el metabolismo social urbano.

En definitiva, este proyecto pretende potenciar el patrimonio cultural intangible del agua (Laureano 1995) a partir de la oportunidad que ofrecen los "caminos del agua" propios de los espacios periurbanos para generar el cambio de visión necesario para una toma de conciencia socio-ecológica, hacia un modelo basado en la autogestión, la autosuficiencia y la producción local. Los recorridos del agua pueden percibirse desde un nuevo camino del agua, que permita leer las relaciones entre la ciudad, la comunidad y el espacio natural desde la percepción del paisaje (Roger 2000) potenciando el cambio de mirada hacia una nueva conciencia comunitaria y productiva, sostenibilista.

\subsection{Metodología}

El proyecto parte de la exploración del pasado desde el estudio del modelo social tradicional (orgánico), atraviesa el presente detectando los conflictos ambientales y sociales del modelo industrial insostenible (post-orgánico) a repensar desde la revalorización del patrimonio y propone un futuro modelo social y cultural re-orgánico (un urbanismo ecológico) de gestión del agua y los espacios de las huertas generando espacios de sociabilidad que conecten con los valores de la sociedad tradicional comunitaria.

Investigando en metodologías participativas es posible incidir en el ámbito político convencional pero sobretodo en las forma y estilos de vida para generar conciencia y pedagogía para la transformación de los marcos perceptivos del paisaje (Lakoff 2007), proponiendo instrumentos que faciliten el cambio de mirada (Cuchí et al. 2010) necesarios para un cambio social y cultural.

El término 'investigación-acción participada' ha sido definido desde múltiples enfoques pero sobre todo desde el ámbito anglosajón. En otras palabras podemos hablar de investigación participada basada en la comunidad (community-based participatory research), investigación dialógica o investigación-acción emancipadora (emancipatory action-research). La forma genérica de nombrar esta metodología de trabajo, la investigación-acción participada (IAP) procede principalmente de las bases de la pedagogía Freireana (Freire 1972). Distintos autores han desarrollado este concepto, especialmente desde los campos de las ciencias sociales. Fals-Borda (1991), White and Pettit (2004) i Ledwith and Springett (2010), son algunos ejemplos.

¿Pero por qué este interés en tomar prestada esta metodología de trabajo dentro del campo del urbanismo ecológico? ¿Cuáles son los retos que supone y cuáles son las ventajas de su propósito?

Desde el punto de vista no participativo, los protocolos que rigen el proceso proyectual en arquitectura y urbanismo -procedan de la administración pública o no- suelen ser demasiado estrictos ya que no dejan margen de flexibilidad para la experimentación en el proceso de proyectar ni a la especificidad ni complejidad de cada lugar o proyecto. Cuando el planeamiento urbano no permite tal oportunidad se hace evidente la necesidad de asegurar otras formas de investigar, otras formas de hacer proyecto y, sobretodo, investigación aplicada en contextos reales. En estas circunstancias, la investigación-acción participada puede ser un buen aliado. 
Para empezar, la IAP persigue la integración del concepto 'participación' con 'investigación', y por tanto, con el proyecto. Según Ledwith and Springett, la clave de la IAP recae en el papel que juega el investigador, en nuestro caso los arquitectos. Éste tratará de minimizar cualquier forma de poder que lo diferencie del resto de agentes vinculados con el proceso de decisión (en nuestro caso: hortelanos/as, usuarios/as de las huertas y ciudadanos/as). El proceso de diseño será por consiguiente flexible, capaz de adaptarse a los cambios que se den. Como arquitectos tenemos el reto de distanciarnos de las capacidades inmediatas de dibujar y cuantificar para implementar otras herramientas como por ejemplo la escucha, la entrevista, la interacción, la mediación y la facilitación de actividades en grupo y los encuentros generales. Margaret Ledwith define así este proceso basado en la empatía y el autoaprendizaje en Participatory practice: community-based action for transformative change:

\footnotetext{
"When the analysis is complete, participants share ideas on how to act on the findings as well as how to evaluate any action taken. This means that those who participate have their knowledge respected, have control over the research process and influence over the way the results are used [...] This ranges from helping people feel confident to develop at their own place, enabling people to come together to share and discuss progress, to ensuring that people who cannot continue still have their voice included in the research in some way". ${ }^{1}$
}

Así pues, el principal propósito consiste en reconocer, identificar y compartir el conocimiento mutuo y las experiencias propias así como alcanzar un grado crítico donde ser capaz de construir nuevos relatos a partir del patrimonio local o el capital intangible del lugar: sus saberes, sus prácticas.

Analizar el lugar desde su pasado, presente y futuro facilita a todos los agentes participantes (nosotras inclusive) la posibilidad de identificar y revalorizar el patrimonio del lugar, comprender otros casos similares, reconocer los saberes en prácticas ecológicas y activar el potencial de decisión para nuevas estrategias de autogestión en el futuro. Además, la posibilidad para un nuevo marco cognitivo sobre el lugar, un cambio de mirada y una transformación de la forma de percibir los valores del lugar.

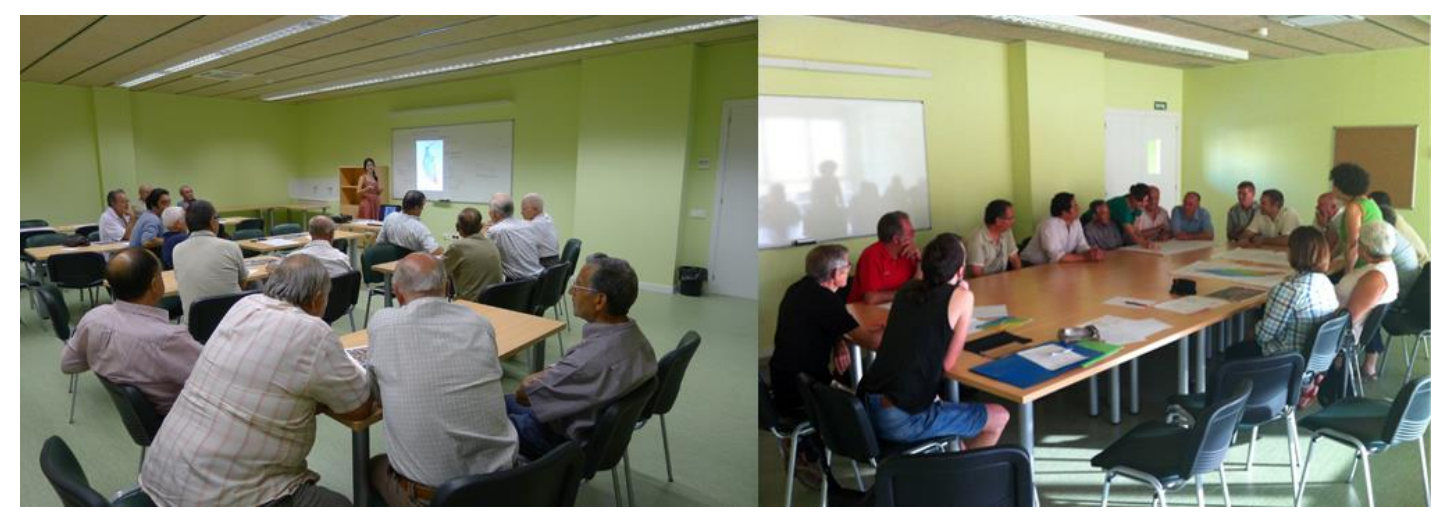

Fig. 3. Montaje del proceso de trabajo de IAP con la comunidad de regantes durante el proyecto. Fuente: Elena Albareda y Marta Serra, 2013.

\footnotetext{
${ }^{1}$ (Ledwith and Springett, 2010:93).
} 


\subsection{Proceso}

El proceso del proyecto ha combinado desde su inicio dos timings y metodologías de trabajo distintos pero complementarios. Por un lado el trabajo comunitario ha estado marcado por reuniones periódicas con los distintos agentes locales, especialmente con la comunidad de regantes como principales usuarios del espacio y conocedores de la gestión del agua. Mientras que por el otro lado, como todo proyecto de espacio urbano, los condicionantes temporales de un proyecto ejecutivo y dirección de obra han precisado dividir la intervención infraestructural en dos fases para su mejor operatividad. La primera fase comprende la construcción de una gran balsa de almacenamiento de agua para retener el flujo constante de agua termal y su enfriamiento. Una vez garantizada su distribución para el abastecimiento de agua de buena calidad y en cantidad suficiente a las huertas, se procederá a la canalización de las aguas negras residuales hasta el colector y a la cubrición de la acequia principal con una pasarela de madera como nuevo acceso peatonal a las huertas.

\subsection{Propuesta final}

Durante el período de un año el proyecto ha pasado por las fases de trabajo de campo, mediación con el ayuntamiento, con la comunidad de hortelanos, una fase de anteproyecto y dos proyectos básicos-ejecutivos para finalmente conseguir los siguientes tres aspectos tanto a escala concreta como general:

En primer lugar, el proyecto propone un plan de mediación y reactivación de la comunidad de hortelanos con el fin de consolidar su estructura, representación y compromiso a la hora de gestionar de forma eficiente el recurso del agua termal.

En segundo lugar, se propone mediante un proyecto ejecutivo de canalización de aguas de saneamiento y cubrición con pasarela en una de las principales acequias, reconvirtiendo y reparando el antiguo torrente que históricamente conducía las aguas de lluvia para el riego y que en la actualidad ha quedado degradado como espacio de vertido directo de aguas grises y negras, adquiriendo la condición de cloaca a cielo abierto. El proyecto propone la canalización de las aguas fecales del saneamiento como si se tratara de un sistema de separativo en régimen seco, separando fecales de pluviales. Además integra la antigua acequia en el entorno de las huertas con la cubrición en forma de pasarela pública de madera, resolviendo problemas de accesibilidad y olores.

Por último, se realiza otro proyecto ejecutivo para la construcción de una balsa de riego. El objetivo de esta intervención es facilitar la acumulación, retención y enfriamiento de las aguas termales sobrantes de los balnearios y lavaderos, manteniendo siempre el sistema de riego actual por tandas, sus acequias y su estructura existente. El proyecto no trata solamente de aportar nuevas fuentes de agua termal sino de hacer más eficiente y sostenible el sistema actual, ahorrando agua que se pierde, re-centralizando las captaciones y fuentes existentes y dotando de espacio donde almacenar para redistribuir.

También se ha optado por construir un vaso de compensación en el punto de desagüe del principal balneario que vertía sus sobrantes hacia el río.

A través del plan de mediación y consenso con los hortelanos, se han priorizado los siguientes aspectos: 
- El recorrido del agua siempre vista, restaurando las acequias existentes, salvo excepciones donde la topografía no lo permite.

- La circulación del agua por gravedad. No se han incorporado dispositivos de bombeo.

- Facilitar la gestión y mantenimiento de la balsa a través del sistema constructivo de hormigón prefabricado escogido por la misma comunidad de regantes. Así se ha evitado el uso de láminas impermeables y se facilita el control visual de llenado y vaciado dejando la balsa al descubierto.

- La integración paisajística del conjunto enterrando la balsa para que su lámina de agua quede a cota rasante del terreno.

- Permitir aislar la balsa del sistema actual por si fuera necesario prescindir de ella. Esto permitiría que la balsa también se pudiera utilizar solamente para el almacenamiento.

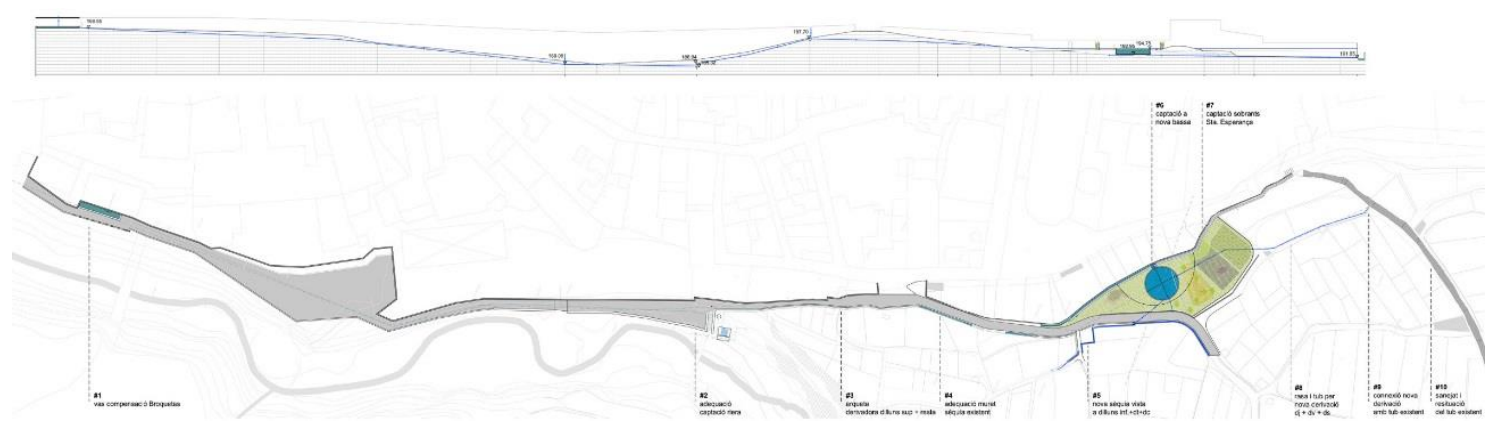

Fig. 4. Planta y sección longitudinal del emplazamiento indicando zonas de intervención. Fuente: CÍCLICA y Jordi Calbetó, 2014.

\section{Resultados y efectos}

El principal efecto generado por el proyecto ha sido el gesto de visibilizar el espacio de las huertas desde la ciudad. Aunque por su configuración física el centro de la ciudad genera una especie de "balcón" a las huertas siendo éstas muy visibles para los ciudadanos, la ciudad ha dado históricamente las espaldas a este espacio agrícola que, progresivamente, ha ido adoptando una condición cada vez más periférica. El hecho de proponer intervenir en las huertas no sólo ha revalorado su presencia en relación al núcleo urbano sino que ha favorecido su conocimiento y sentimiento de apropiación por parte de los vecinos y usuarios. Una apropiación que se hace también evidente en eventos culturales de la ciudad como la Muestra Internacional de Arte Urbano (MIAU) antes citada, que en las últimas ediciones ya ha incorporado las huertas como nuevo escenario de intervenciones artísticas, facilitando así todavía más el sentimiento de apropiación y pertenencia.

Otro gran logro conseguido hasta el momento por el proyecto es la reactivación de la comunidad de regantes, una comunidad que hasta el momento estaba desestructurada, con unos estatutos poco consensuados y sin constitución oficial. El desarrollo participativo del proyecto y las soluciones consensuadas entre la comunidad y el ayuntamiento han facilitado, primero, obtener un registro completo de hortelanos -propietarios y usuarios- y se- 
gundo, que éstos se constituyan como asociación registrada. Reconocerse como comunidad y tener que tomar decisiones conjuntamente por intereses compartidos ha propiciado también que establezcan reuniones quincenales para facilitar la comunicación y toma de decisiones, tanto para el proyecto actual como para el mantenimiento futuro de las infraestructuras construidas y la gestión de la nueva organización de los turnos de riego. Una entidad social de autogestión comunitaria que permitirá la salvaguarda del patrimonio cultural de la gestión del agua facilitando la recogida y transmisión de conocimiento oral necesarios para su perdurabilidad en el tiempo.

\section{Conclusiones: retos, dificultades, aprendizajes}

Nos parece necesario concluir identificando los retos, las dificultades y los aprendizajes que este proyecto ha supuesto para nuestra práctica arquitectónica. Más allá del desarrollo técnico de las propuestas alcanzadas, el proceso de trabajo largo en el tiempo, complejo y diverso en necesidades, intereses y protagonistas, nos obliga a explorar estos espacios 'espejo', estos otros espacios sensibles, hoy tan vigentes y característicos de la revisión de la práctica urbanística y arquitectónica como técnicos profesionales. Abrimos las siguientes líneas de reflexión compartida a modo de conclusión:

Primero: los nuevos roles del arquitecto, el arquitecto como mediador. La figura del arquitecto como etnógrafo parte de la observación de experiencias auto-gestionadas en contextos no occidentalizados, por ejemplo el 'redescubrimiento' durante los años sesenta de las kashbahs o de las barriadas de urbanismo progresivo dentro del período de crisis del Movimiento Moderno. Rod Hackney, Tony Gibson, John Turner, Giancarlo de Carlo o Aldo van Eyck fueron algunos de los arquitectos pioneros dentro del ámbito de la observación y su consiguiente puesta en práctica, la mediación. Así pues, el nuevo papel del arquitecto consistía ya en facilitar los medios técnicos para potenciar la auto-gestión, autoconstrucción y la colaboración o decisiones compartidas. Este tipo de profesional, recuperado hoy día, actúa como catalizador y dinamizador para detonar procesos autónomos que implican una diagnosis, un esfuerzo extra de representación sistémica, la identificación y rearticulación de expertezas locales pero sobretodo la intención de superar los roles de poder propias de cada territorio en las formas de diseño. Las principales dificultades con las que nos hemos encontrado en este tipo de procesos de diseño comunitario son causa de las prioridades instauradas en el sistema dominante: normalmente prima más el resultado como producto que no el proceso como aprendizaje, la rapidez o la obtención de rendimientos cuantificables.

Segundo: la redefinición de la práctica urbanística, hacia un urbanismo socio-ecológico. Si hasta antes de la crisis los proyectos arquitectónicos estaban más motivados por el crecimiento urbano y los beneficios económicos que éste supone, el concepto de viabilidad económica pero también social quedaba relegado a un segundo plano. Actualmente, la creciente conciencia de la amenaza de la huella ecológica generada sobre la biosfera y la necesidad de responder a iniciativas ciudadanas fortalece la responsabilidad ambiental, social y crítica de un nuevo urbanismo, de otras formas de proyectar. Por una parte podemos reconocer este otro urbanismo emergente, informal, proyectado con y desde sus usuarios, basado en la empatía urbana, el sentimiento de comunidad, de identidad y el rol productivo y político del ciudadano empoderado que se viene denominando 'urbanismo táctico' recordando el significado de las tácticas de Henri Lefebvre como formas de apropiación individuales o colectivas que utilizan los ciudadanos en su cotidianidad para ser articuladas de 
forma efectiva en términos políticos, es decir, de transformación de relaciones. Pero por otra parte, a esta dimensión social, le es necesaria sumarle los valores de la sostenibilidad ambiental, constantemente ligados con la relación que establecemos los ciudadanos con nuestro entorno. Si reconocemos esta doble dimensión del nuevo urbanismo, podemos hablar de otra nueva forma de hacer ciudad: el urbanismo socio-ecológico.

Tercero: la necesidad de nuevas herramientas válidas para proyectar este otro urbanismo. No nos sirve proyectar una presentación digital en medio de una reunión entre la comunidad de hortelanos sino que precisamos de hablar del proyecto en el lugar en sí, comprobar y visualizar el tamaño de la balsa a través de un círculo de personas, comprobar el trazado de los nuevos cursos de agua a través de hilos de lana, situando las arquetas con piedras, haciéndolas visibles y móviles, es decir, explicar el lugar desde el lugar mismo. Aun así existen otros ámbitos académicos de los cuales la práctica arquitectónica podría aprender y tomar en préstamos ejercicios y metodologías ya probadas que mejorarían la función del arquitecto como mediador, etnógrafo, facilitador o catalizador. La falta de pedagogía y currículum académico que se imparta dentro de este ámbito dificulta el acceso al arquitecto a instrumentos de trabajo propios de las ciencias sociales como la sociología urbana, la antropología cultural, el trabajo comunitario, la geografía humana, la psicología ambiental, entre otros.

Concluyendo, podemos decir que este proyecto pone en evidencia como uno de los retos que plantea el paradigma de la sostenibilidad es el de recuperar el patrimonio intangible de las gestión de los recursos, más allá del patrimonio físico, como herramienta para la transformación sostenibilista de las ciudades y su relación con el territorio. La dificultad asumida frecuentemente en las disciplinas de la arquitectura y el urbanismo de integrar los procesos participativos, se convierte así en una oportunidad de dotar a los proyectos en zonas patrimoniales de criterios solventes y avalados por el tiempo aportado por la memoria compartida de la comunidad.

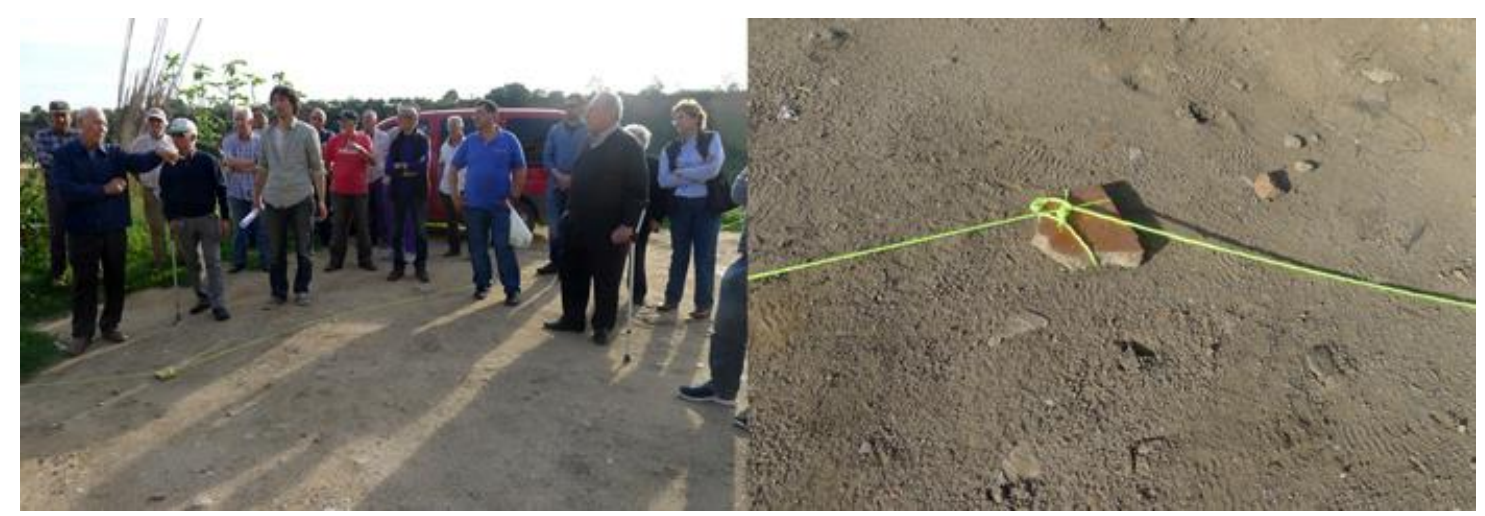

Fig. 5. Montaje del proceso de trabajo de IAP con la comunidad de regantes durante el proyecto. Fuente: CÍCLICA y Jordi Calbetó, 2014.

Agradecimientos. Las autoras de este proyecto agradecemos especialmente a nuestro equipo de trabajo CÍCLICA [space, community \& ecology] colaborando con Jordi Calbetó, arquitecto, a la comunidad de hortelanos y a la concejalía de Sostenibilidad y Espacio Público del Ayuntamiento de Caldes de Montbui por la confianza demostrada en el proyecto. 


\section{Referencias}

Bettini, V. et al. (1996): Elementi di Ecologia Urbana, Torino: Einaudi Editore.

Busquets, J. et al. (2008): Guia d'integració paisatgística 2: Horts urbans i periurbans, Barcelona: Direcció General d'Arquitectura i Paisatge, Generalitat de Catalunya.

Careri, F. (2003): Walkscapes. El andar como práctica estética, Barcelona: Gustavo Gili.

Cuchí, A. et al. (2010): Estrategia Verde Urbana de Santiago de Compostela, Santiago de Compostela.

Certeau, M. de and Giard, L. (1996)[1980]: La Invención de lo cotidiano: I. Artes de hacer, México. D.F.: Universidad Iberoamericana.

Ferreres, V. et al. (2010): Catàleg Mostra Internacional d'Art Urbà: pràctiques artístiques contextuals. Ajuntament Caldes de Montbui, Ajuntament Granollers.

Fischer-Kowalski, M., Huttler, W. (1999): Society's Metabolism. The Intellectual History of Materials Flow Analysis, Part II, 1970-1998, Journal of Industrial Ecology M.I.T., vol 2, 4:107136.

Forman, R., Gordon, M. (1986): Landscape Ecology, New York: John Wiley \& Sons.

Freire, P. (1972): Pedagogy of the Oppressed, Harmondsworth: Penguin.

Guàrdia, M. et al. (2011): La revolució de l'aigua a Barcelona. De la ciutat preindustrial a la metròpoli moderna,1867-1967, Barcelona: MUHBA, Ajuntament de Barcelona.

Hardy and Ward, C. (1984): Arcadia for all: the legacy of a makeshift landscape, London: Mansell.

Hayden, D. (1995): The power of place. Urban landscape as públic history, Cambridge, Massachusetts: The MIT Press.

Lakoff, G., Mora, M. (2007): El cambio de marco es cambio social, in No pienses en un elefante: lenguaje y debate político, Madrid: Editorial Complutense, pp. 4-5.

Laureano, P. (1995): La Piramide rovesciata: il modello dell'oasi per il pianeta terra, Torino: Bollati Boringhieri.

Ledwith, M. and Springett, J. (2010): Participatory practice: community-based action for transformative change, Bristol: The Policy Press.

Lefebvre, H. and Gaviria, M. (1978): El Derecho a la ciudad, 4ª ed. Madrid: Península.

McHarg, I.L. (1969): Design with Nature, New York: Natural History Press.

Mumford, L. (1961): The City in History. Its Origins, Its Transformations and Its Prospects, New York: Harvest Books.

Naredo, J. and Valero, A. (1999): Desarrollo económico y deterioro ecológico, Madrid: Argentaria Visor.

Reclus, E. (2007) [1869]: Histoire d'un ruisseau, Toulouse: Éditions Lume de Carotte.

Roger, A. (2000): Breu tractat del paisatge, Barcelona: La Campana.

Serra, M. and Altés, A. (2010): Re-thinking public space: other públic spaces: community, bybrid collaborative artistic practices and urban reactivation, Barcelona: Secretaria de Joventut de la Generalitat de Catalunya. 
Tello, E. (2006): La Transformación del territorio, antes y después de 1950: un lugar de encuentro transdisciplinar para el estudio del paisaje, AREAS. Revista Internacional de Ciencias Sociales, 25:5-11.

Terradas, J. (2001): Ecologia Urbana, Barcelona: Departament de Medi Ambient.

U.N. United Nations (1993): Earth Summit Agenda 21: The UN Programme of Action from Rio, New York: U.N.

Vidal, A. and Prades, V. (2013): Elogi de l'hort urbà. Vilafranca del Penedès: Edicions i Propostes Culturals Andana, cop.

W.C.E.D. World Commission on Environment and Development (1987): Our Common Future, Oxford: Oxford University Press.

Wrigley, E. (1987): People, Cities and Wealth, Oxford: Blackwell. 